\title{
An Expository on Some Nonstandard Compactifications
}

\author{
Alagu Somasundaram, Rukhmoni Kala \\ Department of Mathematics, Manonmaniam Sundaranar University, Tirunelveli, India \\ Email: alagu391@gmail.com, karthipyi91@yahoo.co.in
}

How to cite this paper: Somasundaram, A. and Kala, R. (2021) An Expository on Some Nonstandard Compactifications. Advances in Pure Mathematics, 11, 807-815. https://doi.org/10.4236/apm.2021.1110054

Received: September 15, 2021

Accepted: October 22, 2021

Published: October 25, 2021

Copyright ( 2021 by author(s) and Scientific Research Publishing Inc. This work is licensed under the Creative Commons Attribution International License (CC BY 4.0).

http://creativecommons.org/licenses/by/4.0/

\begin{abstract}
Three classical compactification procedures are presented with nonstandard flavour. This is to illustrate the applicability of Nonstandard analytic tool to beginners interested in Nonstandard analytic methods. The general procedure is as follows: A suitable equivalence relation is defined on an enlargement ${ }^{*} X$ of the space $X$ which is a completely regular space or a locally compact Hausdorff space or a locally compact Abelian group. Accordingly, every $f$ in $C(X, R)$ (the space of bounded continuous real valued functions on $X)$ or $C_{C}(X, R)$ (the space of continuous real valued functions on $X$ with compact support) or the dual group $\Gamma$ of the locally compact Abelian group $G$ is extended to the set $\bar{X}$ of the above mentioned equivalence classes. A compact topology on $\bar{X}$ is obtained as the weak topology generated by these extensions of $f$. Then $X$ is naturally imbedded densely in $\bar{X}$.
\end{abstract}

\section{Keywords}

Non-Standard, Compactification, Completely Regular Space, Locally

Compact Hausdorff Space, Locally Compact Abelian Group

\section{Introduction}

The foundation stone for Nonstandard analysis was laid by Abraham Robinson in 1966 with the publication of his book "Nonstandard analysis" [1]. Here $R$ is extended to an ordered field extension ${ }^{*} R$ of $R$, where "very small" (infinitesimals [2] [3]) elements and "very large" (infinite) elements exist. Elements of ${ }^{*} R$ are called hyper-real numbers. Sets, relations, functions in $R$ get extended to ${ }^{*} R$ in a natural way. Also statements in $R$ get extended to ${ }^{*} R$, with suitable interpretation, with the aid of a pivotal principle called the Transfer principle. On the other hand, certain statements in ${ }^{*} R$ hold good in $R$ by the Downward 
Transfer principle. The whole idea is not so much to learn the extension as is to study more of the original frame $R$ by getting on a higher platform ${ }^{*} R$. Moreover, the higher platform renders a natural way of looking at the concepts in $R$ and hence provides a natural and shorter proof of even the already proved statements in $R$. Nonstandard analysis propounded by Robinson is not just a formalization of the infinitesimals in $R$. The type of extension from $R$ to ${ }^{*} R$ applies to any mathematical structure based on a suitable nonstandard model. For instance, if $X$ is a topological space or a linear space or a measure space, it gets extended to ${ }^{*} X$ allowing extensions of sets, relations, functions etc., and providing Transfer and Downward Transfer theorems to carry out all algebra and analysis [4]. Again, as in $R$, the idea is to learn more about $X$ and not so much about ${ }^{*} X$.

The purpose of this article is to present nonstandard versions of three classical compactifications: Stone-Cech compactification of a completely regular space, Alexandroff's one-point compactification of a locally compact Hausdorff space and Bohr compactification of a locally compact Abelian group. For the classical versions of Stone-Cech compactification and Alexandroff's one-point compactification and for other preliminaries in topology, we refer to [5] [6] [7]. For Bohr compactification and the preliminaries on locally compact Abelian groups, we refer to [8]. The procedure for nonstandard Stone-Cech compactification is followed for uniform spaces in [9]. This is in analogy with the nonstandard completion of a uniform space mimicking the nonstandard completion procedure of a metric space [4] [10]. Since the basic principle involved is the same in all these, it is contextual to present these in a single article. The general procedure is as indicated in the abstract. These compactifications come under the general category of Q-compactifications [4] for suitable families of functions Q. However, details have to be worked out individually and this has been done systematically in this article.

\section{Main Results}

\section{Definition 2.1.}

Let $X$ be a topological space. A topological space $\bar{X}$ is said to be a compactification of $X$ if $X$ can be embedded in $\bar{X}$ as a dense subspace. That is, if there exists a homeomorphism $\phi: X \rightarrow \bar{X}$ such that range $\phi$ is dense in $\bar{X}$.

First we take up the nonstandard version of the Stone-Cech compactification of a completely regular space. We have the following.

\section{Theorem 2.2.}

Let $X$ be a completely regular space. Then it has a compactification $\bar{X}$.

Proof.

Let $C(X, R)$ be the set of bounded continuous real valued functions on $X$.

In ${ }^{*} X$ define $x^{\prime} \sim y^{\prime}$ if ${ }^{*} f\left(x^{\prime}\right) \simeq{ }^{*} f\left(y^{\prime}\right) \quad \forall f \in C(X, R)$

Clearly $\sim$ is an equivalence relation on ${ }^{*} X$.

We denote the equivalence class of $x^{\prime} \in{ }^{*} X$ by $\left[x^{\prime}\right]$ and the set of equiva- 
lence classes by $\bar{X}$.

$\forall f \in C(X, R)$ we define $\bar{f}: \bar{X} \rightarrow R$ by $\bar{f}\left(\left[x^{\prime}\right]\right)=s t^{*} f\left(x^{\prime}\right)$.

We consider $\bar{X}$ with the weak topology $\omega$ generated by the $\overline{f s}$.

We wish to show that $\varphi: X \rightarrow \bar{X}$ defined by $\phi(x)=[x]$ is an embedding of $X$ as a dense subspace of $\bar{X}$.

(i) First we see that $\varphi$ is one-one.

Let $[x]=[y]$ for $x, y \in X$

Then $x \sim y$

Therefore ${ }^{*} f(x) \simeq{ }^{*} f(y) \quad \forall f \in C(X, R)$

That is, $f(x)=f(y) \quad \forall f \in C(X, R)$

Therefore $x=y$, by complete regularity of $X$

Therefore $\varphi$ is one-one.

(ii) To prove: $\varphi$ is continuous

Let $[y] \in \bar{X}$

A basic neighbourhood of $[y]$ is given by

$$
G=\bigcap_{i=1}^{n}\left\{[z] \in \bar{X}:\left|\bar{f}_{i}([z])-\bar{f}_{i}[y]\right|<\varepsilon\right\}
$$

$\varphi^{-1}(G)=\bigcap_{i=1}^{n}\left\{x \in X:\left|f_{i}(x)-\bar{f}_{i}([y])\right|<\varepsilon\right\}$ is open in $X$, by the continuity of each $f_{i}$.

Hence $\varphi$ is continuous.

(iii) To prove : $\varphi$ is an open map

Let $V$ be a basic neighbourhood of $x \in X$, where $V \in \Psi$

By complete regularity of $X$, fix $f \in C(X, R)$ such that $f: X \rightarrow[0,1]$, $f(x)=0$ and $f\left(V^{c}\right)=\{1\}$

Now $z \in{ }^{*} X,\left.\right|^{*} f(z) \mid<\frac{1}{2} \Rightarrow z \notin\left({ }^{*} V\right)^{c} \Rightarrow z \in{ }^{*} V$

Let $[z] \in \bar{X} \quad$ with $\quad z \in X,|\bar{f}([z])|<\frac{1}{2}$

Then $|f(z)|<\frac{1}{2}$

Therefore $z \in V$, by (1).

Therefore $[z] \in \varphi(V)$

Also $\left\{[z] \in \bar{X}: z \in X,|\bar{f}([z])-\bar{f}([x])|<\frac{1}{2}\right\}$ is a neighbourhood of

$[x]=\varphi(x)$ in $\varphi(X)$

This neighbourhood $=\left\{[z] \in \bar{X}: z \in X,|\bar{f}([z])|<\frac{1}{2}\right\}$,

since $f(x)=0$.

Also this neighbourhood $\subseteq \varphi(V)$, by (2)

Therefore $\varphi(V)$ is open in $\varphi(X)$

Hence $\varphi$ is an open map.

Thus $\varphi: X \rightarrow \bar{X}$ is a homeomorphism of $X$ onto $\varphi(X)$

(iv) To prove: $\varphi(X)$ is dense in $\bar{X}$. 
Let $[y] \in \bar{X}-\varphi(X)$

We take a basic neighbourhood of $[y]$ given by

$$
G=\bigcap_{i=1}^{n}\left\{[z] \in \bar{X}:\left|\bar{f}_{i}([z])-\bar{f}_{i}[y]\right|<\varepsilon\right\}
$$

Now $\bigcap_{i=1}^{n}\left\{x \in{ }^{*} X:\left.\right|^{*} f_{i}(x)-\bar{f}_{i}([y]) \mid<\varepsilon\right\} \neq \phi$, since it contains $y$.

Therefore $\bigcap_{i=1}^{n}\left\{x \in X:\left|f_{i}(x)-\bar{f}_{i}([y])\right|<\varepsilon\right\} \neq \phi$, by Downward Transfer.

That is, $\bigcap_{i=1}^{n}\left\{x \in X:\left|\bar{f}_{i}([x])-\bar{f}_{i}([y])\right|<\varepsilon\right\} \neq \phi$

Therefore $\exists[x] \in G$ for some $x \in X$

Therefore $\varphi(X)$ is dense in $\bar{X}$

(v) To prove: $\bar{X}$ is compact.

For each $[y] \in \bar{X}$, we associate a map $T([y])$ from $C(X, R)$ to $R$ defined by

$$
T[y](f)=\bar{f}([y])=s t^{*} f(y)
$$

Let $A$ be the range of $T$.

Claim: $T$ is a one-one mapping of $\bar{X}$ onto $A$.

$\left[y_{1}\right] \neq\left[y_{2}\right] \Rightarrow{ }^{*} f\left(y_{1}\right)$ and ${ }^{*} f\left(y_{2}\right)$ are not infinitely close to each other for some $f \in C(X, R)$

$$
\begin{aligned}
& \Rightarrow s t^{*} f\left(y_{1}\right) \neq s t^{*} f\left(y_{2}\right) \\
& \Rightarrow \bar{f}\left(\left[y_{1}\right]\right) \neq \bar{f}\left(\left[y_{2}\right]\right) \\
& \Rightarrow T\left(\left[y_{1}\right]\right)(f) \neq T\left(\left[y_{2}\right]\right)(f)
\end{aligned}
$$

Therefore $T\left(\left[y_{1}\right]\right) \neq T\left(\left[y_{2}\right]\right)$

Therefore $T$ is one-one, establishing the claim.

Define a topology on $A$ by declaring $U$ open in $A$ if $T^{-1}(U)$ is open in $\bar{X}$.

By definition, $T$ is a homeomorphism of $\bar{X}$ onto $A$.

To show $\bar{X}$ is compact, all we need to show is that $A$ is compact.

A basic neighbourhood of $\alpha \in A$ is of the form

$$
G=\bigcap_{i=1}^{n}\left\{\beta \in A:\left|\alpha\left(f_{i}\right)-\beta\left(f_{i}\right)\right|<\varepsilon\right\},
$$

where $\varepsilon>0$ and $f_{1}, f_{2}, \cdots, f_{n} \in C(X, R)$

Since $X$ is dense in $\bar{X}, \exists x \in X$ such that $T[x] \in G$

That is, $\left|\alpha\left(f_{i}\right)-T[x]\left(f_{i}\right)\right|<\varepsilon$ for $i=1,2, \cdots, n$

That is, $\left|\alpha\left(f_{i}\right)-f_{i}(x)\right|<\varepsilon$ for $i=1,2, \cdots, n$

That is, $\forall \alpha \in A, \forall f_{1}, \cdots, f_{n} \in C(X, R), \forall \varepsilon>0$ in $R$,

$\exists x \in X$ such that $\left|\alpha\left(f_{i}\right)-f_{i}(x)\right|<\varepsilon$ for $i=1,2, \cdots, n$

By concurrence, $\exists x \in{ }^{*} X$ such that $\forall \alpha \in{ }^{*} A, \forall f \in C(X, R), \forall \varepsilon>0$ in ${ }^{*} R$,

$$
\left|\alpha\left({ }^{*} f\right)-{ }^{*} f(x)\right|<\varepsilon
$$

Taking $\varepsilon>0$ as a positive infinitesimal, we get the following: 
$\exists x \in{ }^{*} X$ such that $\forall \alpha \in{ }^{*} A, \forall f \in C(X, R)$,

$$
\alpha\left({ }^{*} f\right) \simeq{ }^{*} f(x)
$$

Now let $\gamma \in{ }^{*} A$

To show $A$ is compact, we need to show that $\gamma$ is near some $\delta \in A$

By (3), $\exists x \in{ }^{*} X$ such that $\forall f \in C(X, R), \quad \gamma\left({ }^{*} f\right) \simeq{ }^{*} f(x)$

Take $\delta=T([x])$

Then $\forall f \in C(X, R), \delta(f)=T[x](f)=s t^{*} f(x) \simeq{ }^{*} f(x) \simeq \gamma\left({ }^{*} f\right)$

Therefore $\gamma \simeq \delta \in A$

Therefore $A$ is compact.

This completes the proof.

Next in line is Alexandroff's one-point compactification of a locally compact Hausdorff space.

Let $C_{c}(X, R)$ be the set of continuous real valued functions on $X$ with compact support.

Theorem 2.3.

Let $X$ be a non-compact locally compact Hausdorff space. By replacing $C(X, R)$ by $C_{c}(X, R)$ in the proof of last theorem, we get $\bar{X}$ with the weak topology generated by the $\overline{f s}, f \in C_{c}(X, R)$. Then $\bar{X}-X$ is a singleton set and $\bar{X}$ turns out to be the Alexandroff one-point compactification of $X$.

Proof.

In ${ }^{*} X$ define $x^{\prime} \sim y^{\prime}$ if ${ }^{*} f\left(x^{\prime}\right) \simeq^{*} f\left(y^{\prime}\right) \quad \forall f \in C_{c}(X, R)$

Clearly $\sim$ is an equivalence relation on ${ }^{*} X$.

We denote the equivalence class of $x^{\prime} \in{ }^{*} X$ by $\left[x^{\prime}\right]$ and the set of equivalence classes by $\bar{X}, X$ is imbedded in $\bar{X}$ by the mapping $x \rightarrow[x]$ for $x \in X$.

$\forall f \in C_{c}(X, R)$ we define $\bar{f}: \bar{X} \rightarrow R$ by $\bar{f}\left(\left[x^{\prime}\right]\right)=s t^{*} f\left(x^{\prime}\right)$.

We consider $\bar{X}$ with the weak topology $\omega$ generated by the $\bar{f}$.

(i) First we show that $\bar{X}-X \neq \phi$

$\forall x_{1}, x_{2}, \cdots, x_{n} \in X$, take $y \in X$ such that $y \neq x_{i}$ for $i=1,2, \cdots, n$

By local compactness, $\exists f \in C_{c}(X, R)$ such that $f(y) \neq 0$ and $f\left(x_{i}\right)=0$ for $i=1,2, \cdots, n$

Thus $\exists y \in X$ such that $y$ is not related to $x_{i}$ under $\sim$ for $i=1,2, \cdots, n$

By Concurrence, $\exists y^{\prime} \in{ }^{*} X$ such that $y^{\prime}$ is not related to $X$ under $\forall x \in X$

Therefore $\left[y^{\prime}\right] \in \bar{X}-X$ and hence $\bar{X}-X \neq \phi$

(ii) Next we show that $\bar{X}-X$ is singleton

Let $\left[y^{\prime}\right] \in \bar{X}-X$ and $f \in C_{c}(X, R)$ with supp $f=K$, a compact set in $X$.

Then $y^{\prime} \notin{ }^{*} K$; otherwise $y^{\prime} \simeq y$ for some $y \in K$ and $\left[y^{\prime}\right]=[y]$, contradicting $\left[y^{\prime}\right] \in \bar{X}-X$

Therefore ${ }^{*} f\left(y^{\prime}\right)=0$

Thus if $\left[y^{\prime}\right],\left[z^{\prime}\right] \in \bar{X}-X$, then ${ }^{*} f\left(y^{\prime}\right)=0={ }^{*} f\left(z^{\prime}\right) \quad \forall f \in C_{c}(X, R)$

Hence $y^{\prime} \sim z^{\prime}$ 
Therefore $\left[y^{\prime}\right]=\left[z^{\prime}\right]$

Therefore $\bar{X}-X$ is a singleton set, say $\left[x^{\prime}\right]$

Thus $\bar{X}=X \bigcup\left\{\left[x^{\prime}\right]\right\}$, where $X$ is with the identification $x \rightarrow[x]$

From classical analysis, we have $\bar{X}$ is compact with the one-point compactification. Let this topology be denoted by $\mathfrak{I}$.

Now we wish to show $\mathfrak{I}=\omega$

(iii) First we show that every set open in the locally compact Hausdorff space $X$ is open in $\bar{X}$ with the $\omega$-topology.

For this it is enough to show $X$ is open in $\bar{X}$

Take any $x \in X$

$\exists$ an open neighbourhood $U$ of $x$ such that $\bar{U}$ is compact, by local compactness of $X$.

Again we can find an open neighbourhood $V$ of $x$ such that $\bar{V} \subseteq U$.

$\exists f \in C_{c}(X, R), f: X \rightarrow[0,1]$ such that $f(\bar{V})=\{1\}, f\left(U^{c}\right)=\{0\}$.

In particular, $f(x)=1, f\left(U^{c}\right)=\{0\}$.

Since supp $f \subseteq \bar{U}, f \in C_{c}(X, R)$

Then $(\bar{f})^{-1}\left(\left(\frac{1}{2}, 1\right]\right)$ is a $\omega$-open neighbourhood of $[x]$ contained in $X$.

Therefore $X$ is open in $\bar{X}$ and hence any $U$ open in $X$ is open in $\bar{X}$ as well.

(iv) Take any set $K$ compact in $X$.

We show $\bar{X}-K$ is open in $\bar{X}$

Take any $\left[y^{\prime}\right] \in \bar{X}-K$

We need to show that $\left[y^{\prime}\right]$ is an interior point of $\bar{X}-K$

Case $(\mathrm{a}):\left[y^{\prime}\right]=\left[x^{\prime}\right]$

By local compactness, there exists an open set $U$ in $X$ such that $K \subseteq U$ and $\bar{U}$ is compact.

$\exists f \in C(X, R)$ such that $f: X \rightarrow[0,1], f(K)=\{1\}, f\left(U^{c}\right)=\{0\}$

Since supp $f \subseteq \bar{U}, f \in C_{c}(X, R)$

Then $(\bar{f})^{-1}\left(\left[0, \frac{1}{2}\right)\right)$ is a $\omega$-open neighbourhood of $\left[x^{\prime}\right]$ contained in $\bar{X}-K$.

Case (b): $\left[y^{\prime}\right] \in X$

Then $\left[y^{\prime}\right]=[y]$ for some $y \in X-K$

Using local compactness as before, $\exists f \in C_{c}(X, R)$ such that $f: X \rightarrow[0,1], f(y)=0, f(K)=\{1\}$

Then $(\bar{f})^{-1}\left(\left[0, \frac{1}{2}\right)\right)$ is a $\omega$-open neighbourhood of $\left[y^{\prime}\right]=[y]$ contained in $\bar{X}-K$

Therefore in any case $\bar{X}-K$ is open in $\bar{X}$

From (iii) and (iv) we get $\mathfrak{I} \subseteq \omega$

(v) For the other way implication, take any basic open set $(\bar{f})^{-1}(G)$, where $G$ is open in $R$ and $f \in C_{c}(X, R)$

Case (a): Suppose $0 \notin G$ 
Then $(\bar{f})^{-1}(G)$ is $f^{-1}(G)$ with the identification $x \rightarrow[x]$ and so is open $X$ and hence in $\bar{X}$ with the $\mathfrak{I}$-topology.

Case (b): Suppose $0 \in G$

$\frac{\text { Then }(\bar{f})^{-1}(G)}{\{x \in X: f(x) \neq 0\}}=f^{-1}(G) \cup(\bar{X}-K)$, where $K$ is the compact set

Hence $(\bar{f})^{-1}(G)$ is open in $\bar{X}$ with the $\mathfrak{I}$-topology.

Therefore $\omega \subseteq \mathfrak{I}$ and hence $\mathfrak{I}=\omega$

Thus the weak topology generated by the $\overline{f s}$, where $f \in C_{c}(X, R)$, is the Alexandroff one-point compactification of $X$.

Finally we take up Bohr Compactification of a locally compact abelian group.

Let $G$ be a non-compact locally compact abelian group with dual group $\Gamma$. On ${ }^{*} G$ define a relation $\sim$ by $x^{\prime} \sim y^{\prime}$ if ${ }^{*} \gamma\left(x^{\prime}\right) \simeq{ }^{*} \gamma\left(y^{\prime}\right) \quad \forall \gamma \in \Gamma . \sim$ is an equivalence relation on ${ }^{*} G$. Denote the equivalence class of $x^{\prime}$ by $\left[x^{\prime}\right]$ and the set of equivalence classes by $\bar{G}$. Define addition in $\bar{G}$ by $\left[x^{\prime}\right]+\left[y^{\prime}\right]=\left[x^{\prime}+y^{\prime}\right]$. Define $\bar{\gamma}$ on $\bar{G}$ by $\bar{\gamma}\left(\left[x^{\prime}\right]\right)=s t^{*} \gamma\left(x^{\prime}\right)$. Then $\bar{\gamma}$ is well-defined on $\bar{G}$. Topologize $\bar{G}$ by the weak topology generated by the $\bar{\gamma}^{\prime} s, \gamma \in \Gamma$.

\section{Theorem 2.4.}

The above mentioned $\bar{G}$ is a compact abelian group and is a compactification of the locally compact abelian group $G$.

\section{Proof.}

For most part, the proof mimicks that of Theorem 2.2. Since each ${ }^{*} \gamma\left(x^{\prime}\right)$ is finite (in fact $\left|{ }^{*} \gamma\left(x^{\prime}\right)\right|=1$ ), st ${ }^{*} \gamma\left(x^{\prime}\right)$ is defined.

If $x^{\prime} \sim x^{\prime \prime}$, then ${ }^{*} \gamma\left(x^{\prime}\right) \simeq{ }^{*} \gamma\left(x^{\prime \prime}\right)$ and hence $s t^{*} \gamma\left(x^{\prime}\right)=s t^{*} \gamma\left(x^{\prime \prime}\right)$ so that $\bar{\gamma}$ is well-defined.

Clearly $\bar{G}$ is an abelian group.

Next we wish to show that + and - are continuous on $\bar{G} \times \bar{G}$.

Let $\left[x_{\alpha}^{\prime}\right] \rightarrow\left[x^{\prime}\right]$ and $\left[y_{\alpha}^{\prime}\right] \rightarrow\left[y^{\prime}\right]$.

$$
\begin{aligned}
\bar{\gamma}\left(\left[x_{\alpha}^{\prime}\right]+\left[y_{\alpha}^{\prime}\right]\right) & =\bar{\gamma}\left(\left[x_{\alpha}^{\prime}+y_{\alpha}^{\prime}\right]\right) \\
& =s t^{*} \gamma\left(x_{\alpha}^{\prime}+y_{\alpha}^{\prime}\right) \\
& =s t^{*} \gamma\left(x_{\alpha}^{\prime}\right)+s t^{*} \gamma\left(y_{\alpha}^{\prime}\right) \\
& \rightarrow s t^{*} \gamma\left(x^{\prime}\right)+s t^{*} \gamma\left(y^{\prime}\right) \\
& =\bar{\gamma}\left(\left[x^{\prime}\right]\right)+\bar{\gamma}\left(\left[y^{\prime}\right]\right) \\
& =\bar{\gamma}\left(\left[x^{\prime}\right]+\left[y^{\prime}\right]\right)
\end{aligned}
$$

Therefore $\left[x_{\alpha}^{\prime}\right]+\left[y_{\alpha}^{\prime}\right] \rightarrow[x]+[y]$

Therefore + is continuous on $\bar{G} \times \bar{G}$

Similarly - is continuous.

Claim 1: The map $x \rightarrow[x]$ is one to one.

$x \neq y \Rightarrow \gamma(x) \neq \gamma(y)$ for some $\gamma \in \Gamma$, since $\Gamma$ separates points of $G$.

$$
\Rightarrow \bar{\gamma}([x]) \neq \bar{\gamma}([y])
$$

Therefore we have claim 1. 
Claim 2: The map $x \rightarrow[x]$ is continuous.

$$
\begin{gathered}
x_{\alpha} \rightarrow x \Rightarrow \gamma\left(x_{\alpha}\right) \rightarrow \gamma(x) \forall \gamma \in \Gamma \\
\Rightarrow \bar{\gamma}\left(\left[x_{\alpha}\right]\right) \rightarrow \bar{\gamma}([x]) \forall \gamma \in \Gamma
\end{gathered}
$$

Therefore $\left[x_{\alpha}\right] \rightarrow[x]$ in $\bar{G}$

Hence the map $x \rightarrow[x]$ is continuous.

Claim 3: The map $x \rightarrow[x]$ imbeds $G$ as a dense subgroup of $\bar{G}$

Let $[y] \in \bar{G}$

We take a basic neighbourhood of $[y]$ given by

$$
V=\bigcap_{i=1}^{n}\left\{[z] \in \bar{G}:\left|\bar{\gamma}_{i}([z])-\bar{\gamma}_{i}([y])\right|<\varepsilon\right\}
$$

Now $\bigcap_{i=1}^{n}\left\{x \in{ }^{*} G:\left.\right|^{*} \gamma_{i}(x)-\bar{\gamma}_{i}([y]) \mid<\varepsilon\right\} \neq \phi$, since it contains $y$.

Therefore $\bigcap_{i=1}^{n}\left\{x \in G:\left|\gamma_{i}(x)-\bar{\gamma}_{i}([y])\right|<\varepsilon\right\} \neq \phi$, by Downward Transfer.

That is, $\bigcap_{i=1}^{n}\left\{x \in G:\left|\bar{\gamma}_{i}([x])-\bar{\gamma}_{i}([y])\right|<\varepsilon\right\} \neq \phi$

Therefore there exists $[x] \in V$ for some $x \in G$

This establishes claim 3.

Claim 4: $\bar{G}$ is compact.

For each $[y] \in \bar{G}$, associate a map $T([y])$ from $\Gamma$ to $C$ defined by $T[y](\gamma)=\bar{\gamma}([y])=s t^{*} \gamma(y)$

Let $A$ be the range of $T$.

First we see that $T$ is a one-one mapping of $\bar{G}$ onto $A$.

$\left[y_{1}\right] \neq\left[y_{2}\right] \Rightarrow{ }^{*} \gamma\left(y_{1}\right)$ is not infinitely close to ${ }^{*} \gamma\left(y_{2}\right)$ for some $\gamma \in \Gamma$

$$
\begin{aligned}
& \Rightarrow s t^{*} \gamma\left(y_{1}\right) \neq s t^{*} \gamma\left(y_{2}\right) \\
& \Rightarrow \bar{\gamma}\left(\left[y_{1}\right]\right) \neq \bar{\gamma}\left(\left[y_{2}\right]\right) \\
& \Rightarrow T\left[y_{1}\right](\gamma) \neq T\left[y_{2}\right](\gamma)
\end{aligned}
$$

$T$ is one-one.

Now we define a topology on $A$ by declaring $U$ open in $A$ if $T^{-1}(U)$ is open in $\bar{G}$.

By definition, $T$ is a homeomorphism of $\bar{G}$ onto $A$.

To show $\bar{X}$ is compact, it is enough to show that $A$ is compact.

A basic neighbourhood of $\alpha \in A$ is of the form

$V=\bigcap_{i=1}^{n}\left\{\beta \in A:\left|\alpha\left(\gamma_{i}\right)-\beta\left(\gamma_{i}\right)\right|<\varepsilon\right\}$ where $\varepsilon>0$ and $\gamma_{1}, \gamma_{2}, \cdots, \gamma_{n} \in \Gamma$

Since $G$ is dense in $\bar{G}, \exists x \in G$ such that $T[x] \in V$

That is, $\left|\alpha\left(\gamma_{i}\right)-T[x]\left(\gamma_{i}\right)\right|<\varepsilon$ for $i=1,2, \cdots, n$

That is, $\left|\alpha\left(\gamma_{i}\right)-\gamma_{i}(x)\right|<\varepsilon$ for $i=1,2, \cdots, n$

That is, $\forall \alpha \in A, \forall \gamma_{1}, \gamma_{2}, \cdots, \gamma_{n} \in \Gamma, \forall \varepsilon>0$ in $\mathrm{R}, \exists x \in G$ such that $\left|\alpha\left(\gamma_{i}\right)-\gamma_{i}(x)\right|<\varepsilon$ for $i=1,2, \cdots, n$

By concurrence, $\exists x \in{ }^{*} G$ such that $\forall \alpha \in{ }^{*} A, \forall \gamma \in \Gamma, \forall \varepsilon>0$ in ${ }^{*} R$, $\alpha\left({ }^{*} \gamma\right)-{ }^{*} \gamma(x) \mid<\varepsilon$

Taking $\varepsilon>0$ as a positive infinitesimal we get the following:

$\exists x \in{ }^{*} G$ such that $\forall \alpha \in{ }^{*} A, \forall \gamma \in \Gamma, \alpha\left({ }^{*} \gamma\right) \simeq{ }^{*} \gamma(x)$ 
Now let $\beta \in{ }^{*} A$.

To show $A$ is compact, what we need to show is that $\beta$ is near some $\delta \in A$

By $\left.{ }^{*}\right), \exists x \in{ }^{*} G$ such that $\forall \gamma \in \Gamma, \beta\left({ }^{*} \gamma\right) \simeq{ }^{*} \gamma(x)$

Take $\delta=T([x])$

Then $\forall \gamma \in \Gamma, \delta(\gamma)=T[x](\gamma)=s t^{*} \gamma(x) \simeq{ }^{*} \gamma(x) \simeq \beta\left({ }^{*} \gamma\right)$

Therefore $\beta \simeq \delta \in A$

Therefore $A$ is compact.

This completes the proof.

\section{Conflicts of Interest}

The authors declare no conflicts of interest regarding the publication of this paper.

\section{References}

[1] Robinson, A. (1966) Nonstandard Analysis. North Holland Publishing Company, Amsterdam.

[2] Katz, M.G. and Sherry, D. (2013) Leibniz's Infinitesimals: Their Fictionality, Their Modern Implementations, and Their Foes from Berkeley to Russell and Beyond. Erkenntnis, 78, 571-625. https://doi.org/10.1007/s10670-012-9370-y

[3] Blaszczyk, P., Borovik, A., Kanovei, V., Katz, M.G., Kudryk, T., Kutateladze, S.S. and Sherry, D. (2016) A Non-Standard Analysis of a Cultural Icon: The Case of Paul Halmos. Logica Universalis, 10, 393-405. https://doi.org/10.1007/s11787-016-0153-0

[4] Hurd, A.E. and Loeb, P.A. (1985) An Introduction to Nonstandard Real Analysis. Academic Press, Orlando, Florida.

[5] Dugundji, J. (1975) Topology. Prince Hall of India Private Limited, New Delhi.

[6] Munkres, J.R. (2000) Topology A First Course. Prentice Hall of India, New Delhi.

[7] Willard, S. (1998) General Topology. Dover Publications, Inc. Mineola, New York.

[8] Rudin, W. (2017) Fourier Analysis on Groups. Dover Publications Inc., Minneola, New York

[9] Alagu, S. (2021) Nonstandard Compactification of Uniform Spaces. Malaya Journal of Matematik, 9, 882-885. https://doi.org/10.26637/MJM0901/0017

[10] Alagu, S. and Kala, R. (2021) Nonstandard Analysis of Uniform Spaces. Journal of Mathematical and Computational Science, 11, 1053-1062.

https://doi.org/10.28919/jmcs/5277 